\title{
Correspondence
}

\section{SAPHO syndrome in pediatric patients with inflammatory bowel disease treated with infliximab}

\section{Dear Editor}

SAPHO syndrome is a rare disease with bone involvement (aseptic osteitis) and skin manifestations (palm-plantar pustulosis, acne or pustular psoriasis). Due to its subtle clinical presentation, it is often misdiagnosed and unrecognized. The association between SAPHO and inflammatory bowel disease (IBD) has been reported since 1992, with a few pediatric cases described so far [1]. Here we describe two cases of SAPHO syndrome, which occurred in two adolescent girls with Crohn's disease (CD) and Ulcerative colitis (UC), respectively. In contrast to previous reports our patients developed the clinical features of the syndrome during treatment with Infliximab (IFX), despite remission of the underlying IBD.

A Caucasian girl was diagnosed with $C D$ at the age of 7 years due to a complex perianal fistulizing disease. An MRI of the pelvis revealed an inter-sphincter fistula and two peri-anal abscesses. Endoscopic examination and histological findings were consistent with $\mathrm{CD}$. According to current recommendations [2], IFX was started after drainage and fistulectomy, with obtainment of complete remission. After 2 years of maintenance regimen with infusions every 8 weeks, endoscopy revealed mucosal healing. Infliximab was then discontinued and 6-Mercaptopurine was started at a dosage of $1.5 \mathrm{mg} / \mathrm{kg} /$ day. However, after 15 months, IFX was resumed due to $C D$ luminal relapse. With scheduled IFX therapy, she again achieved clinical remission and such treatment was continued for 3 years without need of dose adjustment and with normal growth. At the age of 14, endoscopic examination showed deep remission with mucosal healing. Serum levels of IFX were in the therapeutic range $(14.736 \mathrm{mcg} / \mathrm{ml})$ and anti-IFX antibodies were absent. Fecal calprotectin levels were persistently low at serial measurement. Three months later, 7 weeks after her last IFX infusion, she developed a palm-plantar vesiculopustular rash (Fig. 1a) initially diagnosed as Coxsackie infection. After a few days she developed low-grade fever and marked fatigue. She was admitted to a peripheral hospital where anti-Tumor Necrosis Factor $\alpha$ (antiTNF $\alpha$ ) inducing cutaneous reaction was suspected. Despite local treatment with tazarotene and IFX discontinuation, systemic symptoms did not subside, and additional cutaneous lesions appeared at the trunk and lower extremities. Moreover, the patient started to complain of severe bone pain in multiple sites (jaw, left scapula, lumbar spine, right knees). Thus, she was transferred to our hospital. An infectious disease work-up, including mycobacteria, was negative. Dermatologic findings (Fig. 1a-c) were congruent with anti-TNF $\alpha$ induced reaction, given the concomitance of psoriasiform eczema with secondary alopecia and palm-plantar pustulosis. However, whole-body MRI showed osteitis at clavicle, multiple costae, vertebral bodies, distal femur and proximal tibia bilaterally
(Fig. 2a-b). Bone biopsy confirmed the chronic aseptic inflammatory process, ruling out malignancy or infectious disease. According to the proposed Kahn criteria [3], a diagnosis of active SAPHO syndrome was made. In consideration of the marked involvement of vertebral bodies, treatment with Pamidronate $(1 \mathrm{mg} / \mathrm{kg} /$ day for 3 days) was initiated. Pamidronate was discontinued after the first infusion because of a sharp rise of liver enzymes compatible with drug-induced liver injury. Since Crohn's disease was still in remission (fecal calprotectin level $8 \mathrm{mcg} / \mathrm{g}$; normal intestinal ultrasound) despite active SAPHO syndrome, a switch to Adalimumab (ADA) was attempted at a dosage used for rheumatic conditions ( $24 \mathrm{mg} / \mathrm{mq}^{2}$ every two weeks) in combination with methotrexate (15 mg/m² $\mathrm{m}^{2}$ weekly subcutaneously). Both cutaneous and osteoarticular manifestations dramatically improved with this treatment and she maintains clinical remission and negative inflammation markers at the 12-months follow-up. Total Body MRI showed a marked decrease of bone inflammatory lesions (Fig. 2c).

The second case was a Caucasian girl, diagnosed with UC at 8 years of age. Past medical history was significant only for mild psoriasis, treated with topical medications. Because of pancolitis at disease onset, induction treatment with corticosteroid was successfully adopted and then followed by maintenance treatment with 5-aminosalicylic acid (5-ASA). However, 5-ASA was discontinued after six months due to intolerance and the girl maintained spontaneous remission without therapy for the following two years. At the age of 11, the girl was admitted to hospital for an acute severe attack of UC, unresponsive to corticosteroid, and IFX was started, according to current guidelines [4]. Following IFX therapy, she achieved clinical remission with low fecal calprotectin; thus, azathioprine was added as maintenance treatment. After two months thiopurine-induced leukopenia lead to treatment discontinuation and IFX monotherapy was continued. Serum levels of IFX and anti-IFX antibodies were not available. Endoscopic evaluation after eight months of IFX reported only mild inflammation at cecum and fecal calprotectin was $25 \mathrm{mcg} / \mathrm{g}$, so dose adjustment was not required.

At the age of 12 years (after 16 months of IFX treatment), the patient presented with micro-pustular rash at the trunk with onset 6 weeks after her last IFX infusion: initially a diagnosis of folliculitis was made, and topical erythromycin was started, with only partial response. During the following two weeks, the patient experienced severe bone pain at the spine and hip with antalgic scoliosis. So, she was admitted to our hospital, where a new dermatologic examination documented sterile pustulosis on soles and erythematous-squamous plaques on the scalp and on the trunk. IFX treatment was discontinued and topical therapy with steroid and emollients was started.

A whole-body MRI showed osteitis at several vertebral bodies, sacro-iliac joints, clavicle and sternum. Therefore, she was diagnosed with SAPHO syndrome and treatment with Pamidronate ( $1 \mathrm{mg} / \mathrm{kg} /$ day for 3 days) was started. Due to persistence of bone 


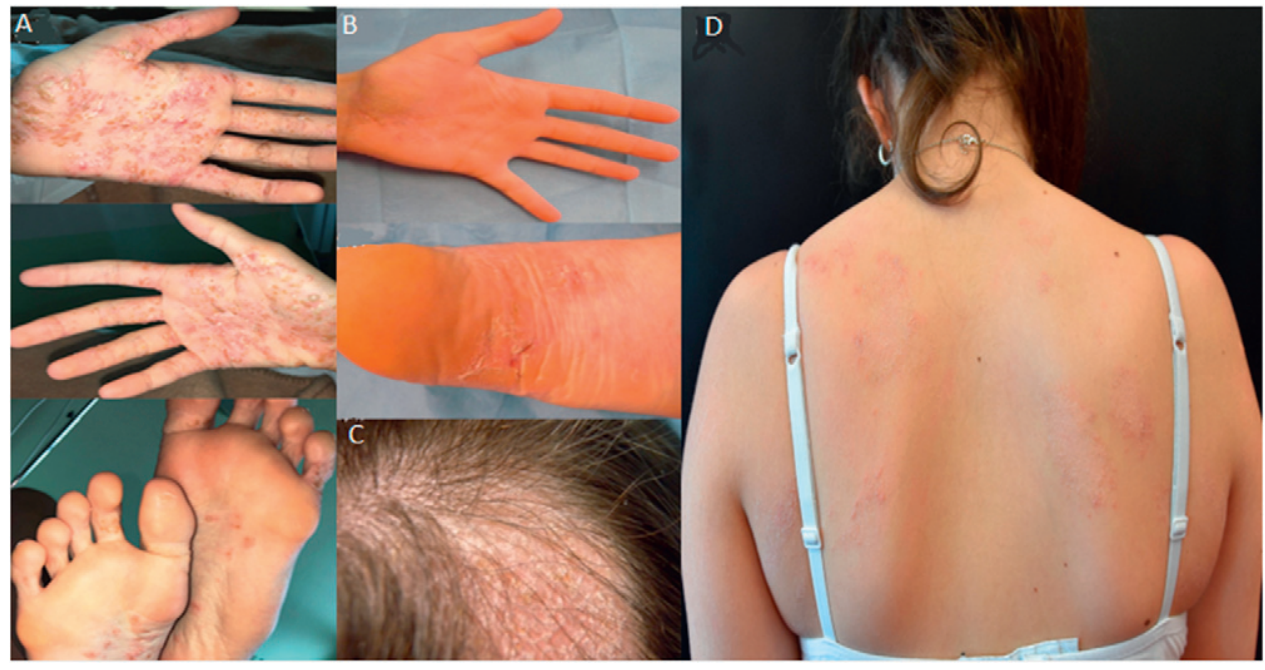

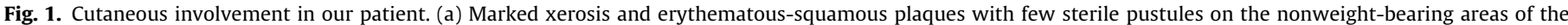

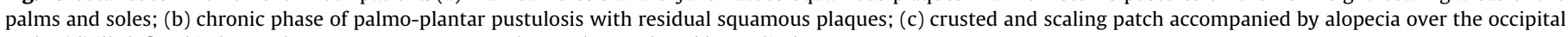
scalp; (d) ill-defined itchy erythematous squamous patches at the trunk and lower limbs.

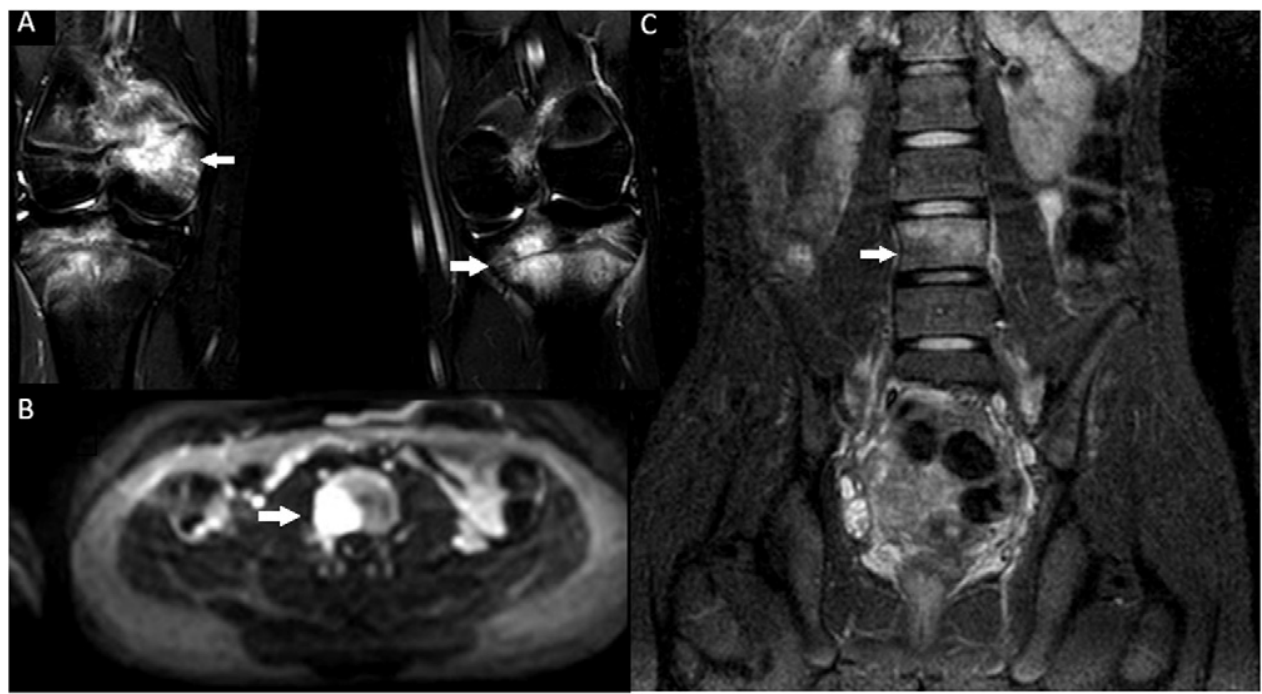

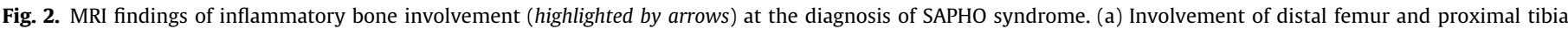
bilaterally. (b) Evidence of osteitis at vertebral body.

pain after treatment, she received ADA $\left(24 \mathrm{mg} / \mathrm{mq}^{2}\right.$ every two weeks) with subsequent improvement of both cutaneous and skeletal symptoms and normalization of inflammatory markers at a 4-months follow-up.

The acronym SAPHO stands for synovitis, acne, pustulosis, hyperostosis and osteitis, thus explaining the prominent features of the disease. Nowadays, this condition is considered part of the spectrum of chronic non-infectious osteomyelitis (CNO), a group of rare multifactorial autoinflammatory disease of the bone characterized by aseptic chronic bone inflammation [3]. The association between SAPHO and IBD have been reported in literature, with an estimated prevalence of around $0.2 \%$ in a cohort of IBD patients and as high as $5 \%$ in patients with a primary diagnosis of SAPHO [1]. An underestimation of the exact prevalence of SAPHO among IBD patients might be due to clinical similarities with some extraintestinal manifestations of IBD and drug-related side effects, such as palmoplantar pustulosis. Palmoplantar psoriasis is indeed frequently associated with anti-TNF $\alpha$ use in IBD patients and it might pose a diagnostic challenge [5].
In SAPHO syndrome anti-TNF $\alpha$ have proven to be effective [6], thus suggesting a crucial role for TNF- $\alpha$ as a mediator in SAPHO pathogenesis. However, our patients developed SAPHO syndrome while under anti TNF $\alpha$ treatment. In addition, in both patients the underlying IBD was in remission and thus this was in contrasts with the reported association between active bowel inflammation and clinical manifestation of SAPHO syndrome [1]. So far, only two cases of SAPHO have been reported during treatment with anti-TNF $\alpha$ : two adult patients with IBD received a diagnosis of SAPHO shortly after starting treatment with IFX and ADA, respectively $[7,8]$. In the latter case, the authors suggested a causal relationship between adalimumab treatment and SAPHO manifestation. In our opinion, the potential triggering role of IFX in these cases is controversial. On one hand, along with palm-plantar pustulosis or pustular psoriasis, two prominent features of SAPHO, the patients presented with xerosis and psoriasiform eczema, which have been recorded as common cutaneous adverse events in patients treated with IFX [9]. On the other hand, the clinical features of SAPHO initially worsened despite IFX discontinuation, while improving when they had been 
given a second anti-TNF $\alpha$ treatment (adalimumab). Moreover, the clinical evolution of our patient is in contrast with the high percentage of recurrence or persistence of the rash in patients with anti-TNF $\alpha$ induced psoriasis and treated with a second anti-TNF $\alpha$ [5]. Indeed, cutaneous adverse events under anti-TNF $\alpha$ are known to be class-specific, since they are related to the inhibition of the physiological function of TNF $\alpha$ in the skin [10]. Therefore, these clues lead us to consider the possibility of SAPHO syndrome onset as a de novo event not related to the concomitant treatment with IFX.

Because of the rarity of the association, treatment of patients with SAPHO and IBD is not standardized. Due to vertebral spine involvement, we preferred bisphosphonate as first line therapy for our patients, in line with current evidence from SAPHO and CNO [3]. Adalimumab was then administered because of reported good response of SAPHO to this agent [3]. However, a low rheumatologic dosage was chosen, considering that IBD was in remission in both patients; in addition, other immunomodulatory agents were given in combination with ADA. Methotrexate was added in the first case in which pamidronate was discontinued due to an adverse effect; in the second patient a course of pamidronate was completed before treatment with ADA.

Hence, SAPHO syndrome should be excluded in IBD patients complaining of skin and osteo-articular symptoms, irrespective of the resolution of intestinal inflammation and simultaneous biologic treatment. Further cases are necessary to elucidate the mechanisms underlying SAPHO during TNF-inhibition and to determine whether a causal link might exist.

Conflict of interest

None declared.

\section{Acknowledgment}

We thanks Roberto Maglie, MD, to have contribuited to clinical data collection and review of paradoxical skin reactions of anti-TNF.

\section{References}

[1] Naves JE, Cabré E, Mañosa M, Grados D, Olivé A, Domènech E. A systematic review of SAPHO syndrome and inflammatory bowel disease association. Dig Dis Sci 2013;58(August (8)):2138-47.

[2] Ruemmele FM, Veres G, Kolho KL, Griffiths A, Levine A, Escher JC, et al. Consensus guidelines of ECCO/ESPGHAN on the medical management of pediatric Crohn's disease. J Crohns Colitis 2014;8(October (10)):1179-207.

[3] Rukavina I. SAPHO syndrome: a review. J Child Orthop 2015;9(February (1)):19-27.

[4] Turner D, Travis SP, Griffiths AM, Ruemmele FM, Levine A, Benchimol EI, et al. Consensus for managing acute severe ulcerative colitis in children: a systematic review and joint statement from ECCO, ESPGHAN, and the Porto IBD Working Group of ESPGHAN. Am J Gastroenterol 2011;106(April (4)):574-88.

[5] Cullen G, Kroshinsky D, Cheifetz AS, Korzenik JR. Psoriasis associated with anti-tumour necrosis factor therapy in inflammatory bowel disease: a new series and a review of 120 cases from the literature. Aliment Pharmacol Ther 2011;34(December (11-12)):1318-27.

[6] Firinu D, Garcia-Larsen V, Manconi PE, Del Giacco SR. SAPHO syndrome: current developments and approaches to clinical treatment. Curr Rheumatol Rep 2016;18(June (6)):35.

[7] Van Den Eynde M, Lecluyse K, Chioccioli C, Brouckaert M, Caussin E, Lammens P. Crohn's disease and the SAPHO syndrome during treatment with infliximab: a case report and review of literature. Gastroenterol Clin Biol 2007;31(June-July (6-7)):607-10.

[8] Amano H, Matsuda R, Shibata T, Takahashi D, Suzuki S. Paradoxical SAPHO syndrome observed during anti-TNF $\alpha$ therapy for Crohn's disease. Biologics 2017;22(May (11)):65-9.

[9] Cleynen I, Van Moerkercke W, Billiet T, Vandecandelaere P, Vande Casteele N, Breynaert C, et al. Characteristics of skin lesions associated with anti-tumor necrosis factor therapy in patients with inflammatory bowel disease. Ann Intern Med 2016;164:10-22.

[10] Segaert H, Hermans C. Clinical signs, pathophysiology and management of cutaneous side effects of anti-tumor necrosis factor agents. Am J Clin Dermatol 2017;18(6):771-87.

\author{
Edoardo Marrani* \\ Gilda Belli \\ Gabriele Simonini \\ University of Studies of Florence, Department of \\ Neurofarba \\ Sandra Trapani \\ University of Studies of Florence, Department of \\ Health Sciences \\ Marzia Caproni \\ University of Studies of Florence, Skin \\ Immunopathology and Rare Dermatological Diseases \\ Unit, 1st Dermatological Clinic P.O. Piero Palagi, USL \\ Toscana Centro, Firenze, Toscana, Italy \\ Paolo Lionetti \\ University of Studies of Florence, Department of \\ Neurofarba \\ * Corresponding author at: Università degli Studi di \\ Firenze, Dipartimento NEUROFARBA, Ospedale \\ Pediatrico Anna Meyer, viale Gaetano Pieraccini, \\ 24, 50139 Firenze, Italy. \\ E-mail address: edoardo.marrani@unifi.it
}

(E. Marrani)

https://doi.org/10.1016/j.dld.2018.09.001

8 July 2018

\section{Allogeneic mesenchymal stromal cells for refractory luminal Crohn's disease: A phase I-II study}

Dear Editor,

Crohn's disease (CD) is a complex chronic relapsing disease involving abnormal systemic and mucosal immune responses against intraluminal antigens, favoured by microbial factors and alteration of the mucosal barrier. A key role in its pathogenesis is played by the imbalance between proinflammatory $\mathrm{T}$ helper (Th) $1 /$ Th 17 cells and regulatory T lymphocytes (Treg), resulting in activation of macrophages and B cells, and recruitment of circulating leukocytes into the gut. Mesenchymal stromal cells (MSCs) exert potent immunomodulatory effects and exhibit interesting properties for tissue repair [1]. Local and intravenous injections of MSCs have demonstrated their safety in fistulising and luminal CD [1]. The efficacy of locally administered MSCs in perianal fistulas associated with $\mathrm{CD}$ has been shown in two randomised controlled trials [2,3], but systemic infusions of MSCs still need to prove their efficacy in luminal CD. Moreover, the mechanisms of action of MSCs in $\mathrm{CD}$ are not fully understood, and the best treatment parameters still need to be determined.

In this pilot phase I-II open-label trial, we aimed to further assess safety and efficacy of intravenous injections of allogeneic bone marrow (BM)-MSCs in severe refractory $C D$ and to characterise blood immune cells during therapy in order to better understand the mechanisms of action of MSCs in CD.

The study protocol conformed to the ethical guidelines of the 1975 Declaration of Helsinki, was approved by the ethics committees of the University Hospital of Liège and the Belgian Federal Agency for Medicines and Health Product, and registered at ClinicalTrials.gov (NCT01540292). Signed informed consent was obtained from participating subjects. Inclusion criteria were age between 18 and 75 years, CD according to LennardJones criteria, clinically and biologically active disease with 